\title{
Evidence of promoting prevention and the early detection of breast cancer among women, a hospital-based education and screening interventions in low- and middle- income countries: a systematic review protocol
}

Adwoa Bemah Bonsu ${ }^{1,2^{*}}$ and Busisiwe Purity Ncama ${ }^{1}$

\begin{abstract}
Background: Given the increasing burden of breast cancer in the low- and middle-income countries, cost-effective approaches are needed to improve the early detection of breast cancer in these continents. Global policies and guidelines are now placing much emphasis on promoting early detection of breast cancer through integrated education and screening interventions. The proposed systematic review aims to map evidence on hospital-based breast cancer education, breast self-examination, and clinical breast examination services for women in low- and middle-income countries.

Methods/design: We will conduct a systematic review of peer-reviewed studies on hospital-based breast cancer prevention intervention (breast cancer education, breast self-examination, and clinical breast examination) for women in low- and middle-income countries. An electronic search will be conducted in the following electronic databases CINAHL Plus with full text (EBSCOhost), MEDLINE with full-text (EBSCOhost) PsychINFO (EBSCOhost), and PubMed. Articles will also be searched through the "Cited by" search and citations included in the reference list of included articles. A two-stage mapping approach will be conducted. The first stage will involve screening studies through assessing their titles. Also, we will screen abstracts of identified studies descriptively and by focus and methods as dictated by the inclusion and exclusion criteria. The second stage will include extraction of data from eligible studies. A parallel screening and data extraction will be conducted by two reviewers. The quality of included studies will be assessed using the mixed methods appraisal tool (MMAT). A narrative account of the data extracted from the included studies will be analyzed using the thematic analysis.

Discussion: We hope to find relevant studies reporting evidence on promoting prevention and the early detection of breast cancer among women in a hospital-based education and screening interventions in low- and middle-income countries. The evidence obtained from the included studies when summarized will help guide future research. The study results will be disseminated electronically and in print. Also, it will be presented at conferences related to breast cancer.
\end{abstract}

Systematic review registration: The protocol has been registered with PROSPERO, with registration number CRD42017077818.

Keywords: Women, Breast cancer, Prevention, Early detection, Education, Breast self-examination, Clinical breastexamination, Screening, Hospital-based, Low- and middle-income countries

\footnotetext{
* Correspondence: bbemahc2000@gmail.com

'Discipline of Nursing, School of Nursing and Public Health, University of KwaZulu-Natal, Durban 4001, South Africa

${ }^{2}$ Department of Nursing, Kwame Nkrumah University of Science and

Technology, Private Mail bag, University Post Office, Kumasi, Ghana
}

(c) The Author(s). 2018 Open Access This article is distributed under the terms of the Creative Commons Attribution 4.0 International License (http://creativecommons.org/licenses/by/4.0/), which permits unrestricted use, distribution, and reproduction in any medium, provided you give appropriate credit to the original author(s) and the source, provide a link to the Creative Commons license, and indicate if changes were made. The Creative Commons Public Domain Dedication waiver (http://creativecommons.org/publicdomain/zero/1.0/) applies to the data made available in this article, unless otherwise stated. 


\section{Background}

Breast cancer $(\mathrm{BC})$ is a malignant growth in any part of the breast and the most common diagnosed cancer among women worldwide [1,2]. Evidence projects that over 19.5 million new cases of $\mathrm{BC}$ will be diagnosed among women globally by the year 2024, of which $55 \%$ will occur in low- and middle-income countries (LMICs) [3]. Studies confirm that the majority of women with BC in most LMICs (50-80\%) report with metastatic disease due to several factors such as inadequate knowledge on $\mathrm{BC}$ and socio-cultural influence on $\mathrm{BC}[4,5]$; resulting in increasing death rate in these communities. As such, the impact of $\mathrm{BC}$ on women, their families and LMICs economy and its resources are substantial, calling for an immediate control and prevention interventions.

Global policies and guidelines suggest prevention as the important priority and the cost-effective approach to curb the world's burden of BC; especially, in LMICs where inadequate trained oncology health personnel, poor infrastructure, and economical and geographical barriers to BC treatment exist [4, 6-9]. World Health Organization [10] recommends screening and early detection as the two main components of cancer prevention/control for countries with high prevalence and mortality rates of cancer as well as late presentation of most curable cancers [10]. The use of active interventions such as breast cancer education (BCE), breast self-examination (BSE), and clinical breast examination $(\mathrm{CBE})$ has shown to decrease the incidence, late presentation, and death rates of $\mathrm{BC}$ among women $[11,12]$. Hence, BCE, BSE, and $\mathrm{CBE}$ seem the effective prevention/control and early detection measures for $\mathrm{BC}$ in limited resource countries [6, 12-14].

CBE and BSE have been strongly endorsed by oncology experts such as the Breast Global Health initiative (BGHI) for LMIC communities due to its contribution to early detection of BC and cost effectiveness [15]. A number of studies suggest that at least BSE improves women literacy and practices on breast in a relatively short time while effective CBE leads to early detection of BC [16-18]. While the benefit of various interventions on $\mathrm{BC}$ prevention/control and early detection for women in most developed countries are well documented [11, 12], research that documents such prevention interventions or enhances early detection of $\mathrm{BC}$ for women in LMICs appears scant. An intervention is considered as a blend of strategic designed program such as educational program and health promotion campaign intended to alter behavior and influence knowledge, beliefs, and attitudes of individuals or improve health status among individuals or an entire population which may be implemented in varied settings [19]. Though there are numerous BC prevention interventions, in this review, $\mathrm{BC}$ prevention intervention will refer to the use of hospital-based BCE, BSE, and CBE services. For this review, $\mathrm{BC}$ prevention and early detection interventions will be explored through the practice of BSE among healthy women with no evidence of malignancy and availability of hospital-based BCE, and CBE services for healthy women with no evidence of cancer in LMICs.

The existing literature indicates that there is a need to adapt effective strategy such as BCE and breast screening that could enhance $\mathrm{BC}$ prevention and early detection $[6,12-14]$. This may significantly reduce incidence and mortality rates associated with $\mathrm{BC}$, facilitate early diagnosis of $\mathrm{BC}$ before metastasis, and improve survival of women with BC in LMICs. This is a systematic review protocol. The rationale of this systematic review will therefore be to map evidence of literature on hospital/ clinic-based $\mathrm{BC}$ prevention and early detection interventions with focus on BCE, BSE, and CBE services for healthy women with no evidence of malignancy in LMICs. The objectives of this systematic review will be as follows:

To review evidence of published literature on existing hospital-based intervention:

To raise women awareness regarding risk factors and early warning signs of breast cancer (e.g., lump in breast)

On BSE practice among healthy women with undetected cancer but, as yet, do not exhibit any symptoms

CBE service for women

The findings from this study will enable the researchers to examine the extent, range, and nature of research activities on hospital-based $\mathrm{BC}$ prevention intervention regarding BCE, BSE, and CBE services for women in LMICs. Additionally, the findings will enable the researchers to identify the existing $\mathrm{BC}$ prevention models and interventions that improve $\mathrm{BC}$ prevention and early detection among women in LMICs.

\section{Methods/design \\ Systematic review}

We will carry out a systematic review of peer-reviewed literature on hospital-based $\mathrm{BC}$ prevention intervention for women in LMICs. A systematic review method was selected as it enhances the mapping of new concepts, types of evidence and gaps related. For the proposed review, we will be guided by preferred reporting items for systematic review and meta-analysis protocols (PRISMA-P) [20]. The framework includes (i) identifying the research question; (ii) identifying the relevant studies; (iii) selecting of studies; (iv) charting the data; and (v) collating, summarizing, and reporting results.

\section{Identifying the research question}

The main research question is what is known from the existing literature about $\mathrm{BC}$ prevention and early detection 
interventions for women in LMICs? The sub-research questions are as follows:

1. What are the existing hospital/clinic-based models for $\mathrm{BC}$ prevention and early detection services in LMICs?

2. What are the available hospital/clinic- based interventions for BCE, BSE and $\mathrm{CBE}$ services for women in LMICs?

\section{Eligibility of research question}

The study will use an amended PICOS (Population, Intervention, Comparison, Outcomes and Study setting) framework to determine the eligibility of the research question as shown in Table 1 below.

\section{Identifying relevant studies}

Primary studies that have a clear empirical base using qualitative, quantitative, and mixed methods published in peer-reviewed journals as well as in gray literature that address the research question will be included. All study designs will be included. An electronic search will be conducted in the following electronic databases CINAHL Plus with full text (EBSCOhost), MEDLINE with full-text (EBSCOhost) PsychINFO (EBSCOhost) and PubMed. Studies will be identified by searching literature that was published in any language from inception. The search terms will be related to women, $\mathrm{BC}$, health care setting, and prevention/early detection intervention. The search strategy will be modified when necessary to suit syntax requirements. Database-specific thesaurus terms (e.g., MeSH terms) as well as free-text terms will be used to search articles. We will implement our search on databases relevant to LMICs that have

Table 1 PICOS framework for determination of eligibility of review question

\begin{tabular}{|c|c|}
\hline Criteria & Determinants \\
\hline Population & $\begin{array}{l}\text { The population of this study will be healthy } \\
\text { women with no evidence of malignancy } \\
\text { accessing a BCE or screening intervention }\end{array}$ \\
\hline Intervention & $\begin{array}{l}\text { Patient-focused BCE and raising awareness } \\
\text { over risk factors, early BC signs and symptoms, } \\
\text { BC screening, and/or detection among women }\end{array}$ \\
\hline Comparison & None \\
\hline \multirow[t]{5}{*}{ Outcomes } & - Increased knowledge on BC \\
\hline & - Practice BSE \\
\hline & - Access CBE services \\
\hline & - Early detection of BC \\
\hline & - Reduced incidence of BC \\
\hline Study setting & $\begin{array}{l}\text { LMICs; within a health care facility } \\
\text { (hospital/clinic) }\end{array}$ \\
\hline
\end{tabular}

been put together in a collaborative effort by Cochrane Groups. To limit our database topic search to studies related to LMICs, a LMICs filter, based on the World Bank classification country list of low-income economies and lower middle-income economies will be employed in proposed databases to retrieve relevant studies. After searching, the studies will be screened against the inclusion and exclusion criteria. Articles will also be searched through the "Cited by" search, and citations included in the reference list of included articles will also be hand searched to identify relevant studies. We used the preferred reporting items for systematic review and meta-analysis protocols (PRISMA-P) as a guide to develop the protocol. Also, the PRISMA-P checklist has been completed to enhance the quality of the protocol [20]. The study protocol has been registered with PROSPERO, with registration number CRD42017077818.

\section{Study selection}

The eligibility criteria were developed to ensure that studies included contain the specific information needed to address the research question on the BC prevention and early detection interventions for women in LMICs.

\section{Eligibility \\ Inclusion criteria}

Studies meeting the following criteria will be included:

- Setting: studies conducted in LMICs within a health care facility. (In this review, health care facility is defined as health care that is based in hospital or clinic).

- Population: healthy women with no evidence of malignancy accessing a $\mathrm{BCE}$ or screening intervention.

- All study types will be included.

- Published studies from inception to date.

- Patient-focused BCE and raising awareness over risk factors, early $\mathrm{BC}$ signs and symptoms, $\mathrm{BC}$ screening, and/or detection among healthy women.

- Outcome: study outcomes will be conceptualized in accordance with the four category frameworks proposed by Harderman and colleagues [23]. This include determinant of behavior (e.g., increased women knowledge), behavioral outcome (e.g., BSE practice and accessing CBE services), physiological and biochemical outcomes (identification of women with pre-malignant disease), and health outcomes (incidence rates and stage of $\mathrm{BC}$ presentation). 
Exclusion criteria

Studies will be excluded if they meet the following characteristics:

- Interventions that solely sought to educate health care professionals on BC awareness or did not specifically include healthy women will be excluded.

- Interventions that solely sought to educate women diagnosed with breast cancer will be excluded.

- Studies that report on community-based interventions will be excluded.

- Studies that will be reported as abstract will be excluded from the review.

The search strategies will be piloted to check the appropriateness of the selected databases and key words. Articles will be searched from the databases by one reviewer who will export all eligible studies into EndNote X7.0.2 reference management software. EndNote X7.0.2 program will be used to check for duplication of articles and to delete the duplicated articles. The reviewer will share the Endnote library with the second reviewer after a comprehensive title screening guided by the criteria for eligibility. Table 2 below illustrates how the electronic data search will be recorded.

Abstracts and full articles of the included studies will be independently screened for eligibility by two reviewers to identify study analysis and assessment. Where there is no agreement between the two reviewers, the studies will be passed on to a third reviewer for consideration. We will seek for help from the University of KwaZulu-Natal (UKZN) library services for articles that are difficult to access. We will also write to the authors to ask for papers in cases of difficult to find articles. Table 3 below presents the results of the pilot search.

The study selection procedure will also be summarized using a PRISMA chart as indicated in Fig. 1 below.

\section{Charting the data}

An analytical method will be employed by two reviewers to extract the background information and process-oriented information of each included study. A data charting form will be developed using Google forms. The variables and themes to include in answering the question will be determined as indicated in Table 4. The data charting form will be

Table 2 Electronic search record

\begin{tabular}{llll}
\hline Date Keyword search & $\begin{array}{l}\text { Search } \\
\text { engine used }\end{array}$ & $\begin{array}{l}\text { Number of } \\
\text { publication } \\
\text { retrieved }\end{array}$ & $\begin{array}{l}\text { Number of } \\
\text { publication after } \\
\text { title screening }\end{array}$ \\
\hline
\end{tabular}

Table 3 Results of the pilot database search

\begin{tabular}{|c|c|c|c|}
\hline $\begin{array}{l}\text { Keyword } \\
\text { search }\end{array}$ & $\begin{array}{l}\text { Date of } \\
\text { search }\end{array}$ & $\begin{array}{l}\text { Search } \\
\text { engine } \\
\text { used }\end{array}$ & $\begin{array}{l}\text { Number of } \\
\text { publications } \\
\text { retrieved }\end{array}$ \\
\hline $\begin{array}{l}\left(\left(\left(\left(\left({ }^{\prime} \text { Breast Neoplasms"[Mesh]) }\right.\right.\right.\right.\right. \\
\text { AND women) AND ((low and } \\
\text { middle income countries)))) } \\
\text { AND (((((((symptoms) OR warning } \\
\text { signs) OR detect*) OR diagnos*) } \\
\text { OR health promotion) OR prevent*)) } \\
\text { OR intervention*)) AND } \\
((((((((((((((((\text { general practitioner*) } \\
\text { OR oncologist) OR physician } \\
\text { assistant) OR physician assisstant) } \\
\text { OR physician) OR clinician) OR } \\
\text { doctor) OR nurse assistant) OR } \\
\text { OR "midwifery"[All Fields] OR } \\
\text { "midwife"[All Fields])) OR midwife) } \\
\text { OR nurse) OR breast clinic) OR } \\
\text { palliative care clinic) OR oncology } \\
\text { clinic) OR clinic) OR } \\
\text { health centre) OR hospital) }\end{array}$ & $\begin{array}{l}\text { September } \\
23,2017\end{array}$ & PubMed & 31 \\
\hline
\end{tabular}

constantly updated. Differences in data extracted will be resolved through discussion among the reviewers and if consensus is not reach, the project lead will be consulted.

\section{Collating, summarizing, and reporting of results}

The aim of this study is to map the existing evidence and to summarize the findings as presented across studies. A narrative account of the data extracted from the included studies will be analyzed using the thematic analysis. Data will be extracted and described around the following outcomes: determinant of behavior (e.g., increased women knowledge), behavioral outcome (e.g., BSE practice and accessing CBE services), physiological and biochemical outcomes (identification of women with pre-malignant disease), and health outcomes (incidence rates and stage of $\mathrm{BC}$ ). Emerging themes will also be coded. NVIVO software version 10 will be utilized collectively to code the data from the included studies based on the above categories [21]. The below process will be followed:

- Coding data from the included articles

- Categorizing the codes into major themes

- Displaying the data

- Identifying key patterns in the data and identify subthemes

- Summarizing

\section{Synthesis}

We will use the resulting themes and assess their relationship to the research question. The reviewers will also analyze the meanings of the findings 


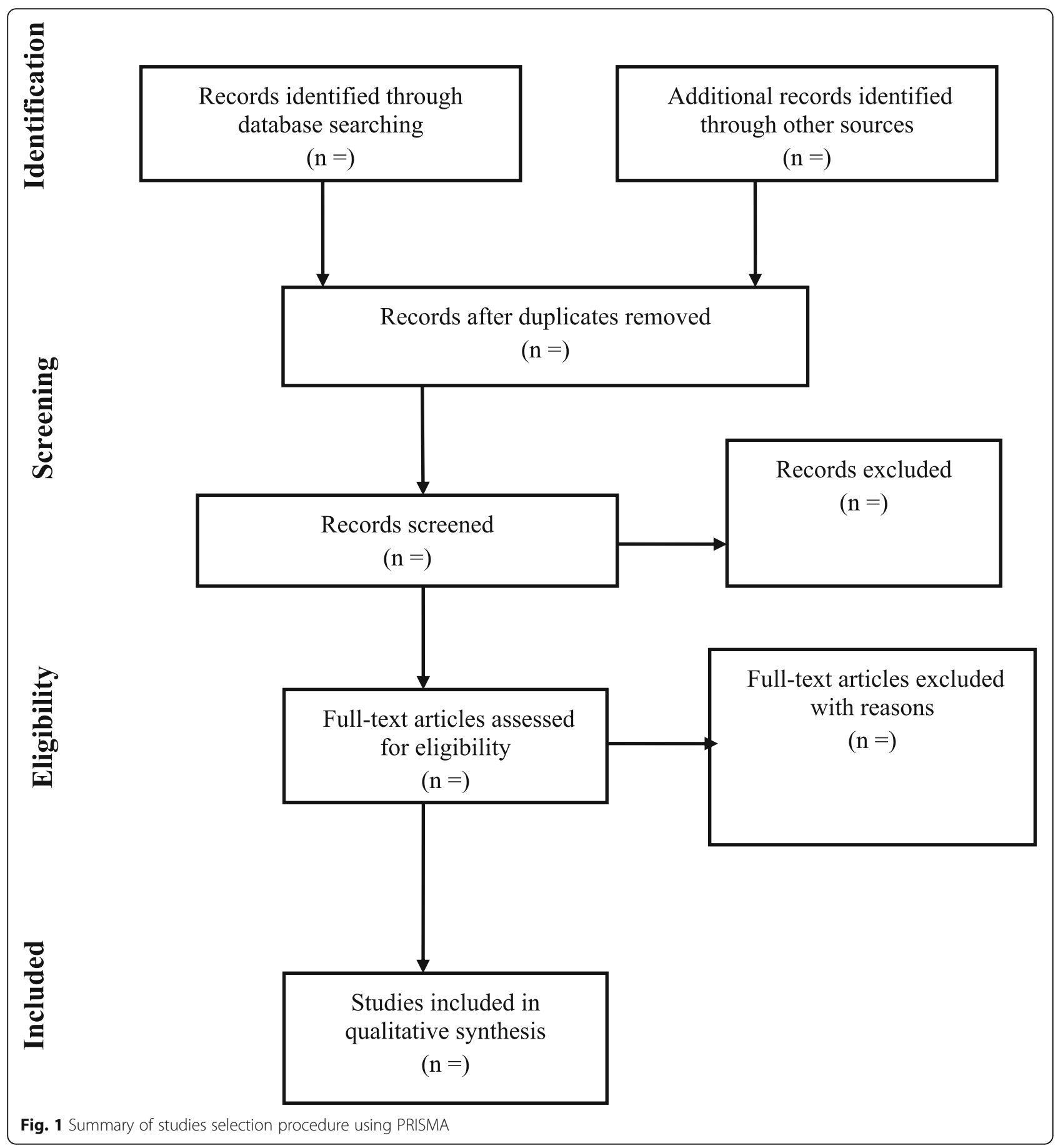

relative to the aim of the study and the significance of these results for future research, policy, and practice.

\section{Quality appraisal}

Quality appraisal is a priority in systematic reviews or part of the systematic review methodology [20]. To enhance the methodological quality of this review, the quality of the studies will be assessed through study appraisal employing the mixed method appraisal tool (MMAT)-Version 2011 [22]. The tool will be used to assess the appropriateness of the aim of the study, adequacy and methodology, study design, participant recruitment, data collection, data analysis, presentation of findings, authors' discussions, and conclusions. This will help in the determination of the quality of the articles using the aforementioned domains. 
Table 4 Data charting (extraction) form

Author and date
Study title
Journal full reference
Study aims
Study design
Population characteristics
Study setting
Recruitment setting
Sampling method
Sample size
Data collection method
Data analysis
Intervention
Outcome (main findings)
Significant outcome (most relevant findings)
Conclusions
Comments

\section{Discussion}

The systematic review will be conducted as a first part of the study on the integration of prevention into cancer palliative care: a case study of breast cancer in a tertiary hospital, Ghana. The review is aimed at mapping the existing evidence and summarizing the findings as presented across the studies on promoting prevention and the early detection of breast cancer for women in LMICs. Additionally, the review will identify the existing hospital/clinic-based education and screening models and interventions that enhance access to BCE, BSE, and CBE services for women in LMICs. Despite that there is a growing recognition that health systems should develop and integrate appropriate, cost-effective cancer prevention, and early detection interventions into existing non-communicable disease (NCD) programs especially in LMICs where most curable cancers are initially diagnosed at an advanced stage [6, 8, 12, 23]; there seems to be paucity of evidence on such models or interventions for $\mathrm{BC}$ for healthy women in LMICs. In order to reduce the incidence and mortality rate of $\mathrm{BC}$, facilitate early detection of $\mathrm{BC}$ among healthy women, and improve survival of women in LMICs, there is a need to explore the $\mathrm{BC}$ education and screening services for women, especially in LMICs $[8,23,24]$. Studies that report on education of health care professionals and women diagnosed with $\mathrm{BC}$ on $\mathrm{BC}$ awareness or did not specifically include healthy women will be excluded because the focus of this review is on healthy women access to $\mathrm{BC}$ education and screening services. Also, studies that report on community-based interventions will be excluded because this review is focused on hospital or clinic-based interventions. Deaths in most women could be avoided if BC education and screening services are available and integrated into NCD programs for healthy women who have no evidence of malignancy. This is because physiologically, women are at risk of BC due to certain specific factors $[25,26]$. The findings of this study may be of interest to relevant stakeholders especially in LMICs where the burden of $\mathrm{BC}$ is significantly high; stakeholders involved in the provision of $\mathrm{BC}$ services, as well as stakeholders advocating for the integration of $\mathrm{BC}$ prevention and early detection services into existing health care systems. In addition, the findings of this study will be of interest to researchers by highlighting gaps in evidence that may require further investigation.

\section{Plans to disseminate study results}

The study results will be disseminated electronically through publications. Further, the results will be disseminated in print to policy makers and stakeholders involved in the provision of $\mathrm{BC}$ services especially in LMICs as well as stakeholders advocating for the integration of $\mathrm{BC}$ early detection services into existing health care systems. Also, it will be presented at conferences related to breast cancer.

\section{Abbreviations}

BC: Breast cancer; BCE: Breast cancer education; BGHI: Breast Global Health Initiative; BSE: Breast self-examination; CBE: Clinical breast-examination; LMICs: Low-middle income countries; MMAT: Mixed method appraisal tool; NCD: Non-communicable diseases; PICOS: Population, Intervention, Comparison, Outcome, Study setting; UKZN: University of KwaZulu-Natal

\section{Acknowledgements}

Not applicable

Funding

Not applicable

Availability of data and materials

All data generated or analyzed during this study will be included in the published systematic review article.

\section{Author's contributions}

$A B B$ and $B P N$ conceptualized and designed the study. ABB prepared the draft under the supervision of BPN. Both ABB and BPN contributed to the development of the background and planned output, methods regarding the review and synthesis of data including sifting and extraction process. ABB prepared the manuscript, and BPN reviewed it. Both ABB and BPN contributed to the reviewed draft version of the manuscript and approved the final version

Ethics approval and consent to participate Not applicable

Consent for publication

Not applicable

Competing interests

The authors declare that they have no competing interests. 


\section{Publisher's Note}

Springer Nature remains neutral with regard to jurisdictional claims in published maps and institutional affiliations.

Received: 3 January 2018 Accepted: 20 November 2018

Published online: 14 December 2018

\section{References}

1. Siu AL. Screening for breast cancer: U.S. preventive services task force recommendation statement. Ann Intern Med. 2016;164(4):279-96.

2. Mukhtar RA, Wong JM, Esserman LJ. Preventing overdiagnosis and overtreatment: just the next step in the evolution of breast cancer care. J Natl Compr Cancer Netw. 2015;13(6):737-43.

3. Anderson BO. UICC World Cancer Congress 2014: global breast cancer trends. In: Washington: the breast health global initiative; 2014. p. 1-10.

4. Obrist M, Osei-Bonsu E, Awuah B, Watanabe-Galloway S, Merajver SD, Schmid K, Soliman AS. Factors related to incomplete treatment of breast cancer in Kumasi, Ghana. The Breast. 2014;23(6):821-8.

5. Ohene-Yeboah M, Adjei E. Breast cancer in Kumasi, Ghana. Ghana medical journal. 2012:46(1)

6. Bosu WK, Cornelison TL, Heckman-Stoddard BM. Breast and gynecologic cancer prevention in low-resource countries. In: Breast and Gynecological Cancers. edn.: Springer; 2013;169-88

7. World Health Organization. Cancer country profiles 2014. Geneva: World Health Organization; 2014.

8. World Health Organization. Cancer control: knowledge into action: WHO guide for effective programmes, vol. 1: World Health Organization; 2006.

9. Anderson BO, Cazap E, El Saghir NS, Yip C-H, Khaled HM, Otero IV, Adebamowo CA, Badwe RA, Harford JB. Optimisation of breast cancer management in low-resource and middle-resource countries: executive summary of the Breast Health Global Initiative consensus, 2010. The lancet oncology. 2011;12(4):387-98.

10. World Health Organization. Cancer control: knowledge into action. WHO guide for effective programmes. Early detection. Geneva: World Health Organization; 2007.

11. Smith RA, Caleffi M, Albert US, Chen TH, Duffy SW, Franceschi D, Nyström L. Breast cancer in limited-resource countries: early detection and access to care. Breast J. 2006;12(s1).

12. Anderson BO, Shyyan R, Eniu A, Smith R, Yip C, Bese N, Chow L, Masood S, Ramsey S, Carlson R: Breast cancer in limited-resource countries: an overview of the Breast Health Global Initiative 2005 guidelines. Breast Journal 2006;12:S3-15.

13. Colditz GA, Kaphingst KA, Hankinson SE, Rosner B. Family history and risk of breast cancer: nurses' health study. Breast Cancer Res Treat. 2012;133(3):1097-104

14. Key T, Appleby P, Barnes I, Reeves G. Endogenous sex hormones and breast cancer in postmenopausal women: reanalysis of nine prospective studies. J Natl Cancer Inst. 2002;94(8):606-16.

15. El Saghir NS, Adebamowo CA, Anderson BO, Carlson RW, Bird PA, Corbex M, Badwe RA, Bushnaq MA, Eniu A, Gralow JR. Breast cancer management in low resource countries (LRCs): consensus statement from the Breast Health Global Initiative. Breast. 2011;20:S3-S11.

16. Shalini DV, Nayak M. Awareness and impact of education on breast self examination among college going girls. Indian journal of palliative care. 2011;17(2):150

17. Moodi M, Mood MB, Sharifirad GR, Shahnazi H, Sharifzadeh G. Evaluation of breast self-examination program using Health Belief Model in female students. Journal of research in medical sciences: the official journal of Isfahan University of Medical Sciences. 2011;16(3):316.

18. Shepherd J, Mclnerney P. Knowledge of breast self-examination in women in Sierra Leone. Curationis. 2007;30(4):38-44.

19. Miller WR, Meyers RJ, Tonigan JS. Engaging the unmotivated in treatment for alcohol problems: a comparison of three strategies for intervention through family members. J Consult Clin Psychol. 1999;67(5):688.

20. Moher D, Shamseer L, Clark M, Ghersi D, Liberati A, Petticrew M, Shekelle P, Stewart LAJSr. Preferred reporting items for systematic review and meta-analysis protocols (PRISMA-P) 2015 statement. 2015; 4(1):1

21. Hardeman W, Sutton S, Griffin S, Johnston M, White A, Wareham NJ, Kinmonth AL. A causal modelling approach to the development of theory- based behaviour change programmes for trial evaluation. Health education research 2005, 20(6):676-687.

22. Pluye P, Robert E, Cargo M, Bartlett G, O'Cathain A, Griffiths F, Boardman F, Gagnon M, Rousseau M. Mixed methods appraisal tool (MMAT) version 2011. Proposal: a mixed methods appraisal tool for systematic mixed studies reviews. 2011. McGill University, Department of Family Medicine, Montreal, Canada.

23. Gelband H, Sloan FA. Cancer control opportunities in low-and middleincome countries: National Academies Press; 2007

24. Harford JB. Breast-cancer early detection in low-income and middle-income countries: do what you can versus one size fits all. The lancet oncology 2011, 12(3):306-12.

25. Okobia M, Bunker C, Zmuda J, Kammerer C, Vogel V, Uche E, Anyanwu S, Ezeome E, Ferrell R, Kuller L: Case-control study of risk factors for breast cancer in Nigerian women. International journal of cancer 2006, 119(9): 2179-85.

26. Bampoe AJ. Risk Factors for Breast Cancer among Patients in Korle-Bu Teaching Hospital A Case-Control. University of Ghana; 2010
Ready to submit your research? Choose BMC and benefit from:

- fast, convenient online submission

- thorough peer review by experienced researchers in your field

- rapid publication on acceptance

- support for research data, including large and complex data types

- gold Open Access which fosters wider collaboration and increased citations

- maximum visibility for your research: over $100 \mathrm{M}$ website views per year

At $\mathrm{BMC}$, research is always in progress.

Learn more biomedcentral.com/submissions 